\title{
Looking into the Historical Warning of Prohibition of Moxibustion at ST36 From Xiao Er (Little Child)
}

\author{
Tong Zheng Hong* \\ As-You-Wish Healthcare Institute, Taiwan
}

Received: 阱 October 22, 2018; Published: 制 October 25, 2018

*Corresponding author: Tong Zheng Hong, As-You-Wish Healthcare Institute, MS in Acupuncture awarded by National University of Health Sciences in IL, Taiwan

\begin{abstract}
ST36 is the most commonly used acupoint for tonification. The warning Prohibition of moxibustion at ST 36 from Xiao Er (little child) is firstly presented in Lei Jing Tu Yi in 1624 and has been discussed for more than three hundred years, but up to now there has not been any detailed information to verify it. Following TCM theories this study presents some explanations like moxibustion at ST36 may damage eyesight and digestion, including metabolism and scientific evidence. In addition, factors like the dosage of moxa, the quality and patterns of patients should be taken into consideration for research in the future.
\end{abstract}

Keywords: Ying Yang; moxibustion; ST36; Shao Yang

\section{Introduction}

In general, most people in Taiwan present themselves to the medical doctors first when they are in need of medical treatments. Part of this fact is because there are not sufficient scientific researches to prove the efficacy of the traditional Chinese medicine (TCM) and acupuncture, including moxibustion, herbs, and cupping. Compared to the current acupuncture developments in Taiwan, acupuncture has gained more and more popularity in the past 40 years and been considered "essential health benefits" in the US and the Western countries [1]. The theories of Ying Yang, Zang Fu, the Five Element, and pattern identification in the TCM are unique and absolutely distinguishes TCM from the Western medicine. Among the TCM theories, the Yin-Yang balance is the most important and the unique concept indicating the harmony of internal organs and the dominating key of the TCM, which has served as the foundation and the guide for the explanation of etiology of diseases and treatments throughout the history of Chinese medicine for more than twenty centuries [1]. It is believed that this Yin-Yang balance explains a disease results from the loss of the balance of Yin Yang. Since diseases are understood to be a loss of balance between Yin and Yang as shown in Figure 1 good results cannot be expected without the positive consideration of Yin and Yang in clinic [1,2]. Furthermore, acupuncture and herbs, two common modalities of TCM in clinic, follow Yin-Yang balance to treat health issues, which have been practiced in the Chinese community for more than 2 millennia.

\begin{tabular}{|cc|}
\hline Yin & Yang \\
\hline & \\
Blood & Qi \\
Material & Function \\
Zang & Fu \\
Yin meridian & Yang meridian \\
Figure 1: Characteristics of Yin and Yang.
\end{tabular}

The most important key to gaining the expected results is that an experienced TCM practitioner or acupuncturist must rely on four skills with meridian identification to make accurate diagnosis and identify precisely the patterns to write up the prescriptions. The warning Prohibition of moxibustion at ST 36 from Xiao Er (小 兒, little child) is firstly presented in Lei Jing Tu Yi (類經圖翼, Illustrated Supplement to the Classic of Categories, 1624) [3] and has been discussed for more than three hundred years in TCM history and the reason still remains uncertain. This study tries to understand if this warning deserves attention from the TCM aspect and possible scientific explanation. 


\section{Age Related Debates in the Warning}

Xu Shen (許慎, 58 - $148 \mathrm{CE}$ ), a Chinese scholar-official and philologist of the Eastern Han Dynasty (25-189), was the author of Shuowen Iiezi (說文解字, Explaining Graphs and Analyzing Characters). Master $\mathrm{Xu}$ in this book provided the information on the development and historical usage of Chinese characters and reported there were a total of 9,353 characters [4]. The brief introduction above to the development of the Chinese characters suggests that definitions of characters vary with time. Definitions of Xiao (小, little) in TCM classics are presented in Table, indicating the difficulty in understanding the accurate age this warning refers to. Judging from the definitions presented above, we can understand age six may be the feasible bound for Xiao. In other words, Xiao Er in Chinese seems to refer to child under six.

Table 1: Definitions of Xiao Er (小兒, little child).

\begin{tabular}{|c|c|}
\hline Name of Classic & Definitions \\
\hline $\begin{array}{c}\text { You Xin Shu (幼幼新書, A New Book of } \\
\text { Pediatrics, 1150) }\end{array}$ & $\begin{array}{c}\text { Over six, Xiao (little); over } \\
\text { sixteen, Shao (teenager) }\end{array}$ \\
\hline $\begin{array}{c}\text { Vol. 1-On disease (卷一, 原病論) You } \\
\text { Ke Fa Hui (幼科發揮, An Expounding of } \\
\text { Pediatrics, 1549) }\end{array}$ & $\begin{array}{c}\text { Under age three, Xiao } \\
\text { (infant) }\end{array}$ \\
\hline $\begin{array}{c}\text { Vol. 8- Overview of Pediatrics (卷八, 兒 } \\
\text { 科總論) Shou Shi Bao Yuan (壽世保元, } \\
\text { Achieving Longevity by Guarding the } \\
\text { Source, 1615) }\end{array}$ & Age 5 or 6, Xiao \\
\hline
\end{tabular}

\section{Glance at Foot Yangming Meridian and ST 36}

Table 2: Functions and indications of ST36.

\begin{tabular}{|c|c|}
\hline \multirow{4}{*}{ Functions } & Tonify deficient Qi a/or Blood. \\
\cline { 2 - 3 } & Tonify Wei Qi and Qi overall \\
\cline { 2 - 3 } Indications & $\begin{array}{c}\text { Earth as the mother of Metal } \\
\text { Gastric pain, vomiting, dysphagia, abdominal distention, } \\
\text { borborygmus, }\end{array}$ \\
\cline { 2 - 3 } & $\begin{array}{c}\text { diarrhea, indigestion, dysentery, constipation, abdominal } \\
\text { pain, acute mastitis }\end{array}$ \\
\cline { 2 - 3 } & $\begin{array}{c}\text { Emaciation due to general deficiency, palpitation, } \\
\text { shortness of breath, poor appetite, lassitude, }\end{array}$ \\
\cline { 2 - 3 } & dizziness, insomnia \\
\cline { 2 - 3 } & Cough and asthma \\
\cline { 2 - 3 } & $\begin{array}{c}\text { Pain in the knee joint, apoplexy, hemiplegia, beriberi, } \\
\text { edema }\end{array}$ \\
\cline { 2 - 3 } & Depressive psychosis and madness \\
\hline
\end{tabular}

The flow route of the Stomach meridian of Foot Yangming shows it begins from the side of the nose at LI 20 (Yingxiang,) and ascends alongside the nose to meet the opposite branch at the bridge of the nose. Then it goes into the inner canthus to meet Bladder meridian of Foot Taiyang at BL 1 (Jinming). Descends downwards connecting CV24 (Chengjiang), ST5 (Daying), GB3 (Shangguan) to the dorsum of the foot at ST42 (Chongyang), runs forwards into the medial side of the great toe at SP1 (Yinbai) to connect with the Spleen meridian
Foot Taiyin. ST36 (Zusanli, 足三里) is the $36^{\text {th }}$ acupoint of the Foot Yangming meridian, located on the anterior aspect of the lower leg, 3 cun below ST 35 (Dubi), and one finger-breadth from the anterior crest of the tibia. Classified as the He-Sea point and the lower He Sea point of the Stomach Meridian, this acupoint is also included in Gao Wu Command Point and Ma Dan-yang Heavenly Star Point [5]. In addition to previous reports with evidence showing it can enhance cell proliferation and neuronal differentiation, affect the limbic and paralimbic systems in the brain that may affect the body's response to stress [6-8], ST36 is commonly recommended and traditionally used in clinic with the features of Command point of the Abdomen, Point of the Sea of Water and Grain for gastrointestinal discomforts [9] with its functions and indications shown in Table 2.

\section{Quick Look at Moxibustion}

Moxibustion, the use of moxa or ai ye in Chinese to warm up regions and meridian acupoints with the intention of stimulating circulation through the points and inducing a smoother flow of blood and qi, is one of the traditional Chinese medicine techniques that can facilitate healing procedure. Moxibustion has been used by the Chinese for thousands of years to strengthen the blood, stimulate the flow of qi, and maintain general health. Recent research has shown moxa can increase blood circulation [10].

It is stated in Spiritual Pivot (Ling Shu, 靈樞) of Yellow Emperor's Inner Classic (Huang Di Nej Jing, 黃帝內經) that where needle cannot work, moxibustion can. Based on both the actions of the meridian system and the roles of moxa and fire that can be warming, the moxibustion has a dual effect of tonification and purgation in TCM theories and is often applied in deficiency-cold syndrome for warming Yang, tonifying/promoting qi, nurturing blood, relieving depletion, dissolving stasis, , dredging channels, and relieving pain, reducing phlegm, eliminating stagnation, removing wind, dispelling dampness, drawing out poison, and purging heat. TCM doctors usually put "needling" and "moxibustion" collectively in practice since both are based on the same theory of meridian and acupoint. In other words, the moxibustion therapeutic effects depend on the meridians and acupoints. The results of scientific researches suggested that moxibustion thermal stimulation can affect both shallow and deep tissues of the skin [11] and the temperature changing of acupoint caused by moxibustion can affect the effects on acupoint and the efficacy of moxibustion [12] In addition to the heat effects the burning moxa can emit visible light and infrared (IR) radiation; therefore, non-thermal radiation effect may be an important role in the efficacy of moxibustion. Debates on the safety of moxa smoke remain unsolved because it is verified that neither volatile nor carbon monoxide would present a safety hazard under normal operating settings; however, some reports show that moxa smoke may be harmful to the human body for causing allergic reactions $[13,14]$. 


\section{Chewing on the Warning}

In ancient TCM classics, it is usually advised that moxibustion at ST36 should be prohibited from children. The most common reason presented in Golden Mirror of Medicine (Yi Zong Jin Jian, 醫宗金鑑) s that the moxibustion at ST36 can be given to adults, but not children with the damage to eyesight. The advice is given in Illustrated Supplement to the Classic of Categories (Lei Jing Tu Yi, 類經圖翼) that a man cannot take moxibustion until age thirty and children are strictly prohibited from moxibustion in order not be sick. In the Volume 39 of Secret of a Frontier Officials it is presented that "He who over age thirty should take moxibustion at ST36, or Qi goes upwards to damage eyes." In this suggestion, we can catch the idea that those who are over thirty need moxibustion at ST36 to prevent the damage to eyes because the effect of moxibustion at ST36 can bring Qi downwards. The concern whether or not this principle can apply to children goes on.

\section{Discussion}

TCM, including acupuncture, and Western Medicine have been viewed as two distinct and divergent medicines for long with the different approaches to physiology and healing techniques. Therefore, the fundamental differences between Western medicine and TCM deserve attention. Constitution is one of the important concepts and concerns in TCM, which highlights the relation of the human beings and the Heaven. In this concept, both the Heaven and humans can interact with each other in providing solutions to the health because TCM sees the human body as a whole and as a microcosm of the universe in diagnosis and treatment, bringing the body, mind and spirit into harmony with Yin Yang balance. On the other hand, "congenital constitution" of the body can result in "root" problems in health. This can scientifically correspond to the core thesis of Precision medicine (PM), which proposes the customization of healthcare with medical decisions, treatments, practices, or products tailored to the individual patient's genetic content. Moxibustion can strengthen the blood circulation and stimulate the flow of qi with its warming property, which represents Yang that can result in uprising Qi and Fire. Ying Yang imbalance leads to discomfort and disease. Channels, properties, indications, and actions of the Chinese herbs in the different material medica classics are not always discussed in the same ways. The actions of Radix Ledebouriellae Divaricatae (Fangfeng) in Compendium of Materia Medica (Bencao Gangmu, 本草綱目), for example, are night sweat, migraine and headache, and constipation. However, actions like aversion to Wind, sweating, blurry vision, and vertigo are presented in The Classic of Herbal Medicine (Shennong Bencaojing, 神農本草經). Licorice root (Gancao, 甘草) is the good example that highlights the time to collect herb is an attention that cannot be ignored. Literature shows the best time to collect and dry Licorice root is in the autumn two to three years after planting [15], but no detailed information is found for the reasons. The possible explanation for harvest time and cultivation time may be much to do with the compound differentiation, which can vary with moisture, temperature, and sunshine. In terms of the physiological development of children, it is stated in the TCM classic Medical mirror that children are pure Yang and Shao (少, little in Chinese) Yang [16]. The meaning of Shao (少) in Chinese is little or few, which indicates that Yang and Qi within children are developing. Normally speaking, physiological condition should develop positively and healthily. In other words, this advice suggests that children should be prohibited from nourishing Yang with moxibustion as Yang in TCM is viewed as energy, function, and rising upwards. On the other hand, excess Yang can consume Ying referring to materials in the body, which can cause Ying-Yang imbalance.

BL1 is the meeting acupoint of the meridians, such as Stomach, Bladder, Small Intestine, Yang Motility, and Ying Motility. The route of the Stomach meridian suggests the use of moxa may make uprising Qi ascend to the eyes, affecting digestion and metabolism with Fire and Heat. One of the functions of ST36 is to tonify "deficient" Qi and/or Blood, which means this deficient condition can be corrected with tonification for Yang and Qi. Moxibustion seems to be adequate for this deficiency, but not for children on the developing stage. Both TCM and acupuncture originated in ancient China and has evolved for more than two thousand years. This fact points out some theories may be presented based on personal experience of TCM masters restricted with geographical factors and transportation. Furthermore, there are not sufficient and detailed information presented in case reports, which makes it harder for TCM and acupuncture researchers and learners to trace back the real reasons.

\section{Conclusion}

The discussion of this warning show there's not enough information for TCM and acupuncture researchers and learners to explore the real reasons and whether or not we should need to follow this warning. However, this warning reminds us that there is a long to go for TCM and acupuncture learners and practitioners to understand the difference between TCM and the Western medicine. Factors like the dosage of moxa, the patterns of the patients, and the time to collect and the processing of moxa, and the treatment during can affect the efficacy, which in truth needs further research.

\section{References}

1. Hong TZ (2017) Exploring a New Extra Point for Sub acute Cough: A Case Report. Scholars Press, Germany.

2. Maciocia G (1989) The foundations of Chinese Medicine. Library of Congress Cataloging in Publication Data, New York.

3. Lei Jing Tu Yi Illustrated Supplement to the Classic of Categories.

4. Hong TZ (2018) Language Barrier before You in TCM and Acupuncture Learning. Advancements Bioequiv Availab 1(3): 515.

5. Deadman P, Al Khafaji M, Baker K (2012) A Manual of Acupuncture. Journal of Chinese Medicine Publications, England.

6. Nam M, Yin CS, Soh K, Choi S (2011) Adult neurogenesis and acupuncture stimulation at ST36. Journal of acupuncture and meridian studies 4(3): 153-158. 
7. Feng Y, Bai L, Ren Y, Wang H, Liu Z, et al. (2011) Investigation of the largescale functional brain networks modulated by acupuncture. Magn Reson Imaging 29(7): 958-965.

8. Pavao TS, Vianna P, Pillat MM, Machado AB, Bauer ME (2010) Acupuncture is effective to attenuate stress and stimulate lymphocyte proliferation in the elderly. Neurosci Lett 484(1): 47-50.

9. Acupressure Point ST36: Stomach 36 or Zu San Li. Explore IM.

10. Moxibustion. Acupuncture today.

11. Huang Q (1989) The combustion temperature of moxa and the temperature change of the body. Foreign Medical Sciences. Traditional Chinese Medicine 11(5): 48.
12. Dong X, Dong Q Xian M (1999) Comparative investigation of effects of different moxibustion methods on temperature of acupoints. Chinese Acupuncture and Moxibustion 19(1): 22-26.

13. Wheeler J, Coppock B, Chen C (2009) Does the burning of moxa (Artemisia vulgaris) in traditional Chinese medicine constitute a health hazard?. Acupuncture in medicine: Journal of the British Medical Acupuncture Society 27(1): 16-20.

14. Li H, Liu S (2008) Cases of moxibustion allergy. Journal of Emergency in Traditional Chinese Medicine; 17(6): 859-860.

15. Hong TZ (2018) Challenges in Learning and Understanding Traditional Chinese Medicine and Acupuncture. Open Acc J Comp \& Alt Med 1(1).

16. Jhong Yi Ji Cheng, The Classic of Medical mirror.

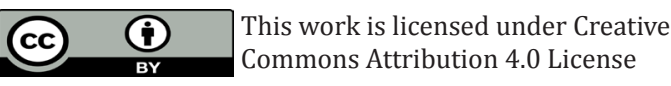

To Submit Your Article Click Here:

Submit Article

DOI: 10.32474/OAJCAM.2018.01.000109

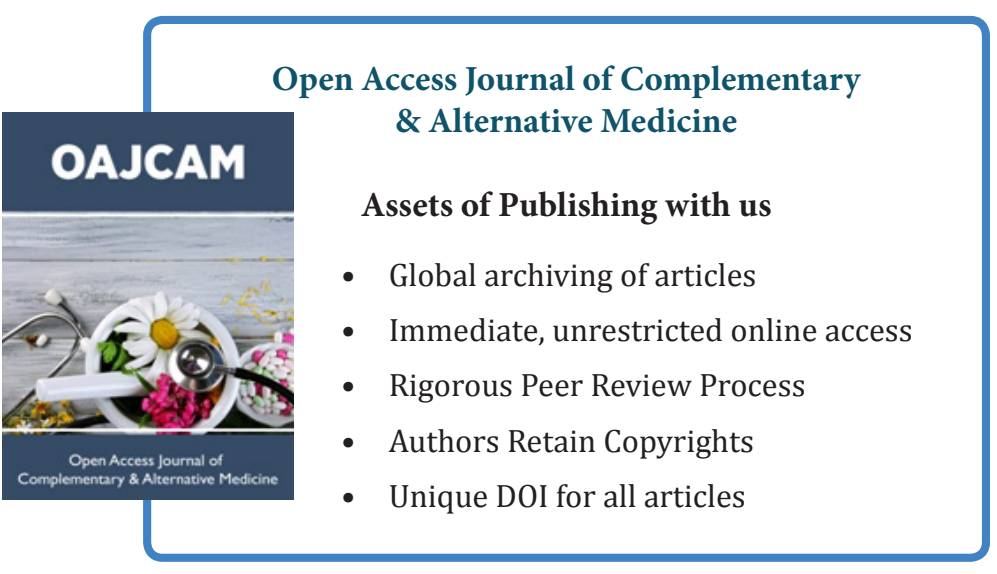

